\title{
Lifetime Trauma History and Cognitive Functioning in Major Depression and Their Role for Cognitive- Behavioral Therapy Outcome
}

\author{
Lena Schindler ${ }^{1}$ (D) , Tobias Stalder ${ }^{2}$ (D) , Clemens Kirschbaum ${ }^{1}$, Franziska Plessow $^{3}$ (D) , \\ Sabine Schönfeld ${ }^{1,4}$, Jürgen Hoyer ${ }^{5}$ (D) , Sebastian Trautmann ${ }^{5,6}$ (D) , Kerstin Weidner ${ }^{7}$, \\ Susann Steudte-Schmiedgen ${ }^{1,7}$
}

[1] Faculty of Psychology, Technische Universität Dresden, Dresden, Germany. [2] Department Erziehungswissenschaften und Psychologie, Universität Siegen, Siegen, Germany. [3] Neuroendocrine Unit, Department of Medicine, Massachusetts General Hospital and Harvard Medical School, Boston, MA, USA. [4] Department of Psychology, Lund University, Lund, Sweden. [5] Institute of Clinical Psychology and Psychotherapy, Technische Universität Dresden, Dresden, Germany. [6] Department of Psychology, Medical School Hamburg, Hamburg, Germany. [7] Department of Psychotherapy and Psychosomatic Medicine, Medical Faculty Carl Gustav Carus, Technische Universität Dresden, Dresden, Germany.

Clinical Psychology in Europe, 2021, Vol. 3(3), Article e4105, https://doi.org/10.32872/cpe.4105

Received: 2020-07-27 • Accepted: 2021-06-03 • Published (VoR): 2021-09-30

Handling Editor: Winfried Rief, Philipps-University of Marburg, Marburg, Germany

Corresponding Author: Susann Steudte-Schmiedgen, Department of Psychotherapy and Psychosomatic Medicine, Medical Faculty Carl Gustav Carus, Technische Universität Dresden, Fetscherstraße 74, 01307 Dresden, Germany. Tel.: +49351458 3634. E-mail: susann.schmiedgen@tu-dresden.de

Supplementary Materials: Materials [see Index of Supplementary Materials]

\begin{abstract}
Background: While cognitive-behavioral therapy (CBT) is the gold-standard psychological treatment for major depression (MD), non-response and lacking stability of treatment gains are persistent issues. Potential factors influencing treatment outcome might be lifetime trauma history and possibly associated primarily prefrontal-cortex- and hippocampus-dependent cognitive alterations.

Method: We investigated MD and healthy control participants with $(\mathrm{MD}+\mathrm{T}+, \mathrm{n}=37$; $\mathrm{MD}-\mathrm{T}+, \mathrm{n}=$ 39) and without lifetime trauma history (MD+T-, $n=26$; MD-T-, $n=45)$ regarding working memory, interference susceptibility, conflict adaptation, and autobiographical memory specificity. Further, MD+T+ $(\mathrm{n}=21)$ and MD+T- groups $(\mathrm{n}=16)$ were re-examined after 25 CBT sessions, with MD-T- individuals $(\mathrm{n}=34)$ invited in parallel in order to explore the stability of cognitive
\end{abstract}


alterations and the predictive value of lifetime trauma history, cognitive functioning, and their interaction for treatment outcome.

Results: On a cross-sectional level, $\mathrm{MD}+\mathrm{T}+$ showed the highest conflict adaptation, but MD+T- the lowest autobiographical memory specificity, while no group differences emerged for working memory and interference susceptibility. Clinical improvement did not differ between groups and cognitive functioning remained stable over CBT. Further, only a singular predictive association of forward digit span, but no other facets of baseline cognitive functioning, lifetime trauma history, or their interaction with treatment outcome emerged.

Discussion: These results indicate differential roles of lifetime trauma history and psychopathology for cognitive functioning in $\mathrm{MD}$, and add to the emerging literature on considering cognitive, next to clinical remission as a relevant treatment outcome.

\section{Keywords}

major depression, lifetime trauma history, working memory, interference susceptibility, conflict adaptation, autobiographical memory, cognitive-behavioral therapy

\section{Highlights}

- Conflict adaptation was highest in MD with lifetime trauma history.

- Autobiographical memory specificity was lowest in MD without lifetime trauma history.

- No differential treatment response was found in MD with and without lifetime trauma history.

- There were no changes of cognitive functioning over CBT, irrespective of lifetime trauma history.

- Only singular predictive value of cognitive functioning for CBT success emerged.

Meta-analyses suggest cognitive-behavioral therapy (CBT) as the gold-standard psychological treatment for major depression (MD; e.g., Barth et al., 2013; Cuijpers et al., 2014), a condition characterized by depressed mood and loss of motivation together with behavioral alterations such as reduced activity and disturbed sleep (Diagnostic and Statistical Manual of Disorders - Fifth Edition; American Psychiatric Association, 2013). However, a substantial patient subgroup fails to achieve clinically significant symptom improvement, with non-response and dropout rates of approximately $34 \%$ and $25 \%$, respectively (for meta-analytic data, see Cuijpers et al., 2014; Hans \& Hiller, 2013). This highlights the need to enhance our understanding of factors associated with psychopathology and treatment outcome, allowing an optimization of CBT effects and reduction of dropout rates. Here, trauma history is frequently discussed, defined as exposure to actual or threatened death, serious injury, or sexual violence (American Psychiatric Association, 2013). Particularly for childhood trauma in MD, associations with poorer therapy response, longer remission time, and greater need for additional medication are relatively well-researched (for review and meta-analytic data, see Nanni et al., 2012; Nemeroff, 
2016; Teicher \& Samson, 2013). Notably, lifetime trauma, including childhood, adulthood, or both types of trauma, has been far less well studied, except for one study suggesting negative associations of both childhood and adulthood adversity with therapy outcome in MD (Miniati et al., 2010).

Importantly, lifetime trauma history is assumed to co-occur with neurobiological (e.g., Kolassa \& Elbert, 2007; Sherin \& Nemeroff, 2011) and cognitive alterations (e.g., Vasterling \& Arditte Hall, 2018). However, data on this and its influence on therapy success in the context of MD and trauma is sparse. In particular, primarily prefrontal-cortex- and hippocampus-dependent functioning have received attention (McIntyre et al., 2013; Rock et al., 2014; Snyder, 2013; Snyder \& Hankin, 2019). Regarding the former, of importance might be working memory (WM) as a facet of executive functioning (EF) relevant for temporal maintenance (usually assessed by the repetition of a list of numbers) and manipulation (usually assessed by the repetition of a list of numbers in a backward fashion) of content necessary for current tasks (Diamond, 2013). Accumulating evidence suggests impaired WM in patients with MD (for reviews, see Snyder, 2013; Snyder \& Hankin, 2019). Further, one study reported childhood trauma to predict performance in a compound WM score of information maintenance and manipulation in both patients with MD and healthy controls (Saleh et al., 2017), but another found no WM differences with respect to information maintenance or manipulation in patients with MD with or without childhood trauma (Dannehl et al., 2017).

An EF domain considered to be even more impaired in MD (e.g., Snyder, 2013; Snyder \& Hankin, 2019) is the ability to suppress irrelevant and/or interfering response tendencies while pursuing mentally represented goals (i.e., inhibitory control, Diamond, 2013). Typically, this is studied via the well-known Simon task (Simon, 1990), where the inhibition of a response following a task-irrelevant visual stimulus is necessary as a different response is required. The resulting additional performance costs (i.e., slower reaction times [RTs] and/or increased percentages of error [PEs]) compared to trials with matching automatic and required tendencies comprise the so-called Simon effect as a measure of interference susceptibility (Simon, 1990). After response conflicts, inhibitory control is typically increased, leading to a decreased impact of task-irrelevant information compared to trials not following conflicts. The resulting difference in the Simon effect is termed conflict adaptation (Botvinick et al., 2001). In MD, particularly this conflict adaptation according to task demands is suggested to be increased (van Steenbergen et al., 2012). Notably, previous work from our group revealed similar findings for patients with posttraumatic stress disorder (PTSD) and, albeit less clearly, trauma-exposed controls (Schindler et al., 2020; Steudte-Schmiedgen et al., 2014), encouraging research on the interaction of trauma and MD.

Of note, there is an abundance of studies suggesting not only EF, but also mainly hippocampally-driven overgeneral memory retrieval (OGM) to be a central correlate of MD (for meta-analytic data, see, e.g., Sumner et al., 2010). This increased recall of over- 
general (e.g., "I am happy when meeting friends") instead of specific autobiographical memories (e.g., "I was happy on July 8 when I met friends"; Williams et al., 2007) is also prevalent in PTSD, with trauma history a potential shared mechanism (Moore \& Zoellner, 2007; Ono et al., 2016; Sumner et al., 2010; Williams et al., 2007). However, previous contrasting of trauma-exposed and non-exposed individuals with MD (notably, again only focusing on childhood trauma) provided mixed results, with one study finding OGM only in trauma-exposed (Aglan et al., 2010) and another only in non-exposed individuals (Kuyken et al., 2006).

Next to these cross-sectional findings of certain alterations of EF and autobiographical memory domains, and the possible mediating role of trauma history in MD, it is plausible to assume that such alterations show significant change over psychotherapy. However, the vast majority of studies could not detect any changes of the cognitive alterations described above over psychotherapy/combined psycho- and pharmacotherapy (for WM, see, e.g., Beblo et al., 1999; Lahr et al., 2007; for inhibitory control, see, e.g., Schmid \& Hammar, 2013; but Ajilchi et al., 2016; for OGM, see, e.g., Peeters et al., 2002). Thus, a current meta-analysis (Bernhardt et al., 2019) rather support the suggestions from previous reviews (e.g., Bernhardt et al., 2019; Köhler et al., 2015; Moore \& Zoellner, 2007; Snyder \& Hankin, 2019) of high stability of such alterations even after clinical remission, with improvements not exceeding task-specific practice effects. While previous data on cognitive markers as predictors for clinical outcome in the context of pharmacotherapy is promising (Groves et al., 2018), research on CBT is outstanding, except for initial studies suggesting a predictive value of enhanced autobiographical memory specificity (Sumner et al., 2010), but not interference susceptibility (Goodkind et al., 2016). However, while lifetime trauma history is assumed to be associated with both therapy outcome (e.g., Nemeroff, 2016; Teicher \& Samson, 2013) and cognitive alterations (e.g., Vasterling \& Arditte Hall, 2018) in MD, a combined investigation is still pending.

Hence, the aim of the current study was to examine (i) lifetime trauma history and (ii) facets of cognitive functioning (i.e., WM, interference susceptibility, conflict adaptation, and OGM) as well as (iii) their interaction in the context of MD symptomatology and therapy success. Due to the inconclusive literature on the interplay of lifetime trauma history and MD for cognitive functioning, our first step was to study respective baseline alterations in $\mathrm{MD}$ and healthy control participants with $(\mathrm{MD}+\mathrm{T}+, n=37$; $\mathrm{MD}-\mathrm{T}+, n=$ $39)^{1}$ and without lifetime trauma history (MD+T-, $n=26$; MD-T-, $n=45$ ). Specifically, we aimed to (1) investigate whether the previously found effect of lifetime trauma history on conflict adaptation (Schindler et al., 2020; Steudte-Schmiedgen et al., 2014) is also visible in MD and (2) shed light on the conflicting evidence regarding OGM (Aglan

1) $\mathrm{MD}+\mathrm{T}+=$ patients with $\mathrm{MD}$ with lifetime trauma history; $\mathrm{MD}+\mathrm{T}-=$ patients with $\mathrm{MD}$ without lifetime trauma history; MD-T+ = patients without MD with lifetime trauma history; MD-T- = patients without MD and without lifetime trauma history. 
et al., 2010; Kuyken et al., 2006). Further, we assessed clinical and cognitive treatment outcome under consideration of lifetime trauma history by re-examining patients with $\mathrm{MD}$ with $(\mathrm{MD}+\mathrm{T}+, n=21)$ and without lifetime trauma history (MD+T-, $n=16)$ after 25 CBT sessions. In order to account for practice effects, non-traumatized healthy control individuals (MD-T-, $n=34$ ) were re-invited in parallel. Here, we hypothesized (3) poorer treatment outcome for $\mathrm{MD}+\mathrm{T}+$ than for $\mathrm{MD}+\mathrm{T}$ - individuals. Based on recent meta-analytic evidence (Bernhardt et al., 2019), we aimed to examine whether we could confirm the finding of (4) no changes of cognitive functioning over CBT, irrespective of lifetime trauma history, also for the tasks studied here. On a last note, we aimed to (5) exploratorily study the predictive value of cognitive functioning for CBT outcome.

\section{Method}

\section{Participants and Procedures}

Recruitment was conducted within the outpatient unit of the Institute of Clinical Psychology and Psychotherapy of the Technische Universität Dresden, as well as via flyers and local advertisements. Individuals were included in the study if they were aged between 18 and 65 years, not pregnant (women), and did not report any severe physical diseases (e.g., cancer, encephalopathy) over the past five years. Further exclusion criteria concerned hair-related and endocrine factors due to biomarker analyses reported elsewhere (e.g., glucocorticoid medication; Steudte et al., 2013; Steudte-Schmiedgen et al., 2014). The presence of MD and any other DSM-IV (American Psychiatric Association, 2007) mental disorders was assessed using the standardized Munich Composite International Diagnostic Interview (DIA-X/M-CIDI; Wittchen \& Pfister, 1997) conducted by therapists of the outpatient unit or trained research team members and confirmed by an experienced clinical psychologist. Twenty-eight participants from the MD groups showed psychiatric comorbidities within the last 12 months (one: $n=15$, two: $n=8$, three or more: $n=5)$. Those encompassed specific $(n=12)$ or social phobia $(n=13)$, somatoform disorders $(n=6)$, panic disorder with or without agoraphobia $(n=8)$, generalized anxiety $(n=3)$, obsessive-compulsive $(n=2)$, adjustment $(n=2)$, or eating disorders $(n=1)$.

An assignment to the MD groups was based on a current primary 12-month MD diagnosis and no 12-month diagnosis of substance abuse or dependence (except for nicotine) or any lifetime diagnoses of psychosis, severe depressive disorder with psychotic symptoms, or bipolar disorder. Notably, individuals meeting the lifetime diagnostic criteria for PTSD were also excluded from the study, in order to allow insights into the role of lifetime trauma exposure per se for cognitive functioning in MD. Participants were included in the control group if they did not report any lifetime mental disorders according to the DIA-X/M-CIDI stem questions and the Mini International Neuropsychiatric Interview (M.I.N.I.; Sheehan et al., 1998). Participants were further classified as exposed or 
non-exposed to lifetime trauma based on the Posttraumatic Stress Diagnostic Scale (PDS; Ehlers, Steil, Winter, \& Foa, 1996). For an allocation to the T+ groups, both the "objective" A1 ("actual or threatened death or serious injury or a threat to the physical integrity of oneself or others") and the "subjective" A2 criterion ("intense fear, helplessness or horror") had to be met, following the DSM-IV requirements that qualify life events as traumatic (American Psychiatric Association, 2007). The control groups are the same as in the parallel study on patients with PTSD (Schindler et al., 2020). For further participant characteristics, see Table 1 and Supplementary Materials (type of lifetime trauma history).

CBT for MD groups was conducted within the outpatient unit based on established manuals (Hautzinger, 1998, 2008) and supervised by experienced therapists. After 25 sessions, $\mathrm{MD}+\mathrm{T}+$ and $\mathrm{MD}+\mathrm{T}-$ patients were re-invited for clinical and cognitive testing, with MD-T- participants being contacted in a parallel fashion (no difference regarding months between assessments: $M=13.5, S D=3.86 ; M=11.56, S D=4.03$; and $M=14.76$, $S D=6.97$, respectively; $\left.F(2,68)=1.78, p=.177, \eta_{\mathrm{p}}^{2}=.05\right)$. Among the 63 patients with MD examined at baseline, 6 (9.5\%) were only interested in the cross-sectional study, 16 (25.4\%) dropped out of CBT, and 41 (65.1\%) completed therapy. Between those who dropped out of CBT and those who did not, no differences emerged regarding pre-treatment clinical variables (all $p s \geq .219$ ). All participants had provided written informed consent before study inclusion. The study protocol was approved by the ethics committee of the Technische Universität Dresden (EK 65022010) and conducted in accordance with the Declaration of Helsinki.

\section{Clinical and Psychological Measures}

Self-developed questionnaires were applied for socio-demographic (age, sex, education status) and health-related variables (smoking, chronic physical diseases, regular medication intake). Depressive symptoms over the previous two weeks were assessed via the Beck Depression Inventory-II (BDI-II, Hautzinger et al., 2006). The PDS (Ehlers et al., 1996) provided insights into the presence or absence of lifetime trauma history and the severity of symptoms associated with posttraumatic stress according to DSM-IV criteria. The Trauma History Questionnaire (THQ, Maercker, 2002) provided an overview over number and frequency of potentially traumatic events fulfilling the DSM-IV A1, but not A2 criterion (Hooper et al., 2011). Furthermore, to obtain information on the severity of childhood maltreatment (irrespective of fulfilling DSM-IV A criteria), the Childhood Trauma Questionnaire (CTQ, Gast et al., 2001) was used. At follow-up, patients with MD additionally received the revised version of the Questionnaire of Changes in Experience and Behavior (Veränderungsfragebogen des Erlebens und Verhaltens VEV-R; Zielke \& Kopf-Mehnert, 2001). This allowed a classification of patient-evaluated therapy effects via 42 items of opposite polarity (e.g., "Compared with the time prior to initiation of 


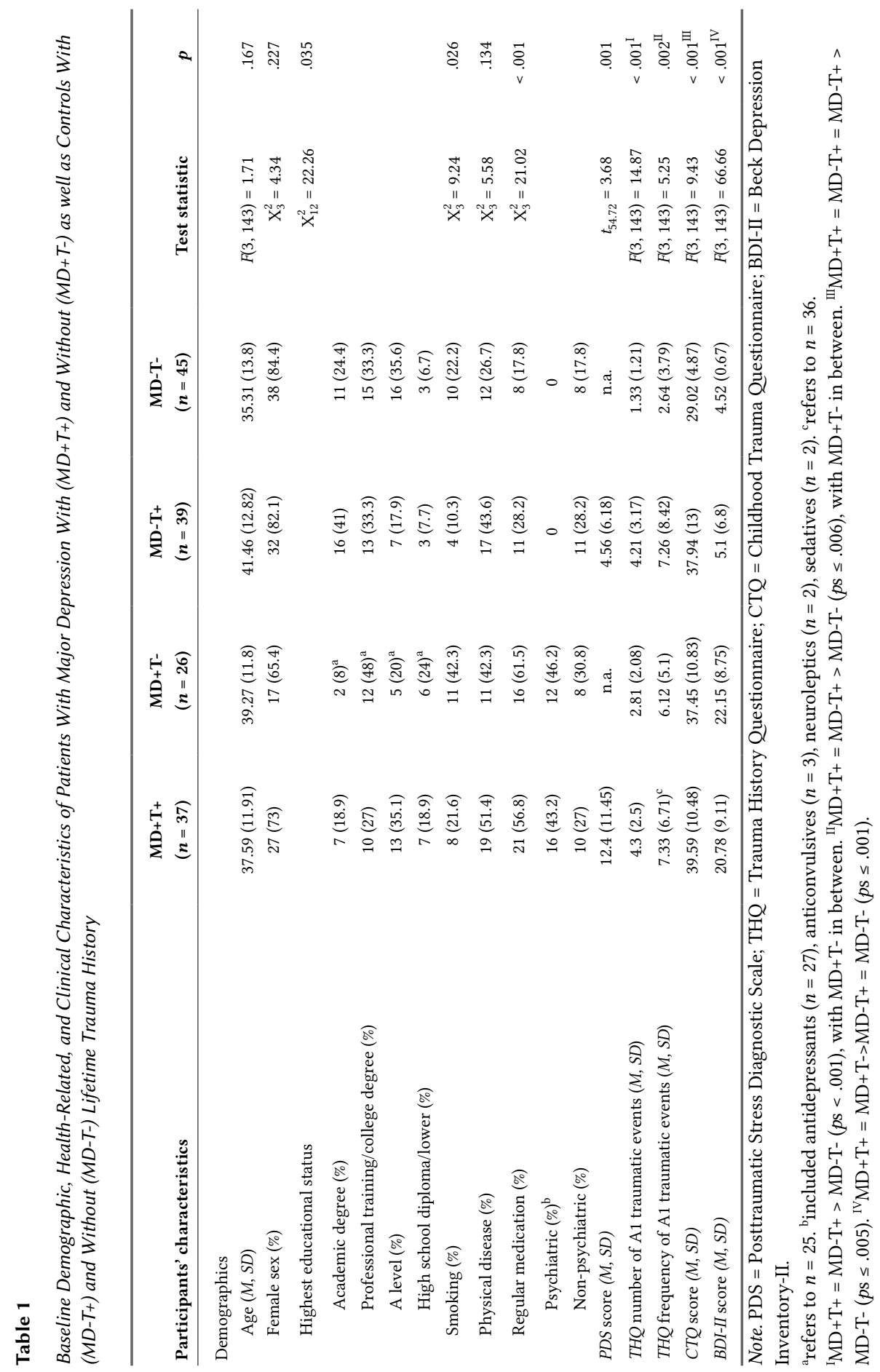


therapy, I feel more relaxed/no change/more tense.") into three categories (i.e., symptom improvement, no change, and worsening).

\section{Cognitive Tasks}

WM was examined using the Wechsler Memory Scale digit span task (Wechsler, 1997). Participants repeated a series of numbers read out loud by the experimenter in a forward (information maintenance) or backward fashion (information manipulation). Interference susceptibility and conflict adaptation were assessed by a number version of the Simon task (Fischer et al., 2008). In brief, participants categorized the numbers 1 to 9, except 5 , as smaller or larger than five by pressing a left (Alt) or right (Alt Gr) key on a QWERTZ keyboard with their left or right index finger, respectively. Although task-irrelevant, stimulus location automatically facilitates the pressing of the corresponding response button, either in accordance, or in conflict with the required action, resulting in compatible and incompatible trials, respectively. The resulting difference in RTs and PEs comprises the Simon, and the typical reduction of interference susceptibility after conflict trials the conflict adaptation effect (Botvinick et al., 2004; Simon, 1990). Participants completed a 16-trial practice, followed by three 64-trial test blocks, resulting in 192 test trials (for further details, see Schindler et al., 2020; Steudte-Schmiedgen et al., 2014). Indices for interference susceptibility $(\mathrm{I}-\mathrm{C})$ and conflict adaptation [( I - cC) - (iI - iC)] (lowercase letters: compatibility of the previous, uppercase letters: compatibility of the current trial, larger values indicating more pronounced effects) were calculated (van Steenbergen et al., 2010).

Autobiographical memory specificity was assessed via the standardized Autobiographical Memory Test (Williams \& Broadbent, 1986). Participants were instructed to read words out loud (practice phase: three neutral words, testing phase: five positive and five negative words in a pseudo-randomized order, starting with a positive word and alternating valence) and briefly describe a related specific autobiographical memory. The words were randomly chosen from a word pool from a previous study (Schönfeld \& Ehlers, 2006) matched for word frequency, emotionality, imagery, and pleasantness (apart from positive words rated as more pleasant than negative ones; Hager \& Hasselhorn, 1994), with different sets used at baseline and follow-up. Answers were tape-recorded, transcribed and coded by trained research assistants. As an outcome variable, the number of specific memories was used, defined as having happened at a particular place and time more than one week ago and having lasted for one day or less. If no answer was provided within 30 seconds, the trial was considered an omission. For assessing inter-rater-reliability, a second, independent rater re-assessed a random sample $(10 \%)$ of the tape-recorded sequences, resulting in $\kappa=.76$ for the baseline and $\kappa=.82$ for the follow-up assessment. 


\section{Statistical Analyses}

Analyses were conducted via SPSS for Windows, version 25 (IBM, Armonk, NY), R (R Core Team, 2017), and STATA 15.1 (StataCorp LLC, 2017). Cross-sectional group comparisons were carried out via univariate analyses of variance (ANOVAs; continuous variables) and $\mathrm{X}^{2}$ contingency tables (dichotomous variables). For the Simon task, the first trial of each block (1.6\%), posterror trials (3\%), target repetitions (11.3\%), and, for RT analyses, error trials (3\%) were excluded. AMT data from one MD+T- and one MD-Tparticipant were missing.

For longitudinal analyses, as a first step, participants from the MD+T+, MD+T-, and MD-T- groups with available longitudinal data were re-examined regarding baseline demographic and clinical differences. Simon task data from one MD+T+ and three MD-Tparticipants, and AMT data from one MD+T- and two MD-T- participants were missing. Again, the first trial of each block (1.6\%), posterror trials (baseline: $2.8 \%$, follow-up: $2.7 \%$ ), target repetitions (baseline: 11.5\%, follow-up: 10.9\%) and, for RT analyses, error trials (baseline: 2.8\%, follow-up: 2.7\%) were excluded. Repeated-measures ANOVAs with time [2, baseline vs. follow-up] as within-subject and group [3, MD+T+ vs. MD+T- vs. MD-T-] as between-subject factor were applied to assess clinical and cognitive changes over CBT.

Exploratory linear/logistic regression analyses were conducted for examining the predictive value of lifetime trauma history ( $P D S$; yes/no) for changes of depressive symptom severity $(B D I-I I)$ and dropout from care as core outcome measures, respectively. Due to the small sample size for the longitudinal analyses, and the high correlations between depressiveness $(B D I-I I)$ and the subjectively evaluated therapy effects ( $V E V-R, r=-.66, p$ $<.001)$ at the follow-up assessment, we decided to omit the $V E V-R$ from the predictive analyses. For the BDI-II, a change score was computed by subtracting baseline from follow-up values, and baseline values were included as a covariate to the regression analyses. As a second step, baseline cognitive performance (centered around the mean to avoid multicollinearity issues), and, as a third, the interaction of lifetime trauma history (yes/no) and baseline cognitive performance were added to the model.

Whenever hypothesis testing referred to one major cognitive domain (i.e., EF and learning/memory) and were not exploratory in nature, Holm-Bonferroni correction (Holm, 1979) for family-wise error (FWER) per respective domain was applied. As the assumptions of conventional GLMs (ANOVA, linear regression) are frequently violated in psychological data possibly leading to poor power and inaccurate effect sizes (Field \& Wilcox, 2017), we repeated hypothesis testing using robust regressions. These drop GLM assumptions by using a robust sandwich estimation of standard errors, down-weighting observations with large residuals, and omitting outlying residuals (Royall, 1986). Predictive analyses were repeated using mixed-effects regressions with random intercept parameter addressing regression to the mean, which can otherwise yield biased results (Oberg \& Mahoney, 2007). However, due to the higher prevalence and familiarity of 
conventional GLMs in the field, whenever both analyses yielded the same results, conventional GLMs were reported.

\section{Results}

\section{Sample Characteristics, Clinical Symptomatology, and Baseline Cognitive Functioning}

The groups were well-matched regarding age, sex, and physical diseases (all $p s \geq .134$, see Table 1). However, group differences emerged for educational status $\left(X_{12}^{2}=22.26\right.$, $p=.035)$ and smoking $\left(\mathrm{X}_{3}^{2}=9.24, p=.026\right)$. Furthermore, both clinical groups reported higher medication intake than the non-clinical ones $\left(\mathrm{X}_{3}^{2}=21.02, p<.001\right)$, mainly driven by psychiatric medication. However, including these variables as covariates did not change the cross-sectional results. For depressive symptom severity (BDI-II), both MD+Tand $\mathrm{MD}+\mathrm{T}+$ individuals reported higher levels than the control groups (all $p \mathrm{~s} \leq .001$ ), with post-hoc analyses indicating no difference between them. For number and frequency of DSM-IV A1 traumatic events, both MD+T+ and MD-T+ scored higher than MD-Tindividuals, with $\mathrm{MD}+\mathrm{T}$ - individuals in between $(T H Q$, all $p s \leq .006)$. For the severity of childhood maltreatment, both MD+ groups as well as the MD-T+ participants scored higher than the MD-T- group $(C T Q$, all $p s \leq .005)$.

No group differences emerged for forward, backward, and overall digit span (all $p \mathrm{~s}$ $\geq .283$, see Table 2). For the Simon task, groups differed regarding conflict adaptation of median RTs with a medium effect size, $F(3,143)=3.23, p=.024, \eta_{\mathrm{p}}^{2}=.063,90 \% \mathrm{CI}[0, .12]$, see Figure 1), with higher levels in MD+T+ compared to MD-T- individuals $(p=.017)$ and no other differences (all $p s \geq .43$ ). Neither for conflict adaptation of mean PEs, nor for interference susceptibility did group differences emerge (all ps $\geq .424$ ). Regarding OGM, for positive and negative words and the overall score, $\mathrm{MD}+\mathrm{T}$ - participants scored lower than both MD-T+ and MD-T- ones with, again, medium effect sizes (all ps $\leq .002$ ), and no other differences (all $p \mathrm{~s} \geq .118$ ).

While OGM results remained stable after Holm-Bonferroni correction for FWER, the group difference for conflict adaptation of median RTs lost statistical significance ( $p=.168$ ). Applying robust regressions did not considerably change the results, except for the difference between MD-T+ and MD-T- participants regarding conflict adaptation of median RTs and the interference effect of median RTs emerging as non-significant trends, $\beta=-15.2,95 \%$ CI $[-30.7,0.2], p=.053$ and $\beta=-11.5,95 \%$ CI $[-24.2,1.2], p=.076)$. 


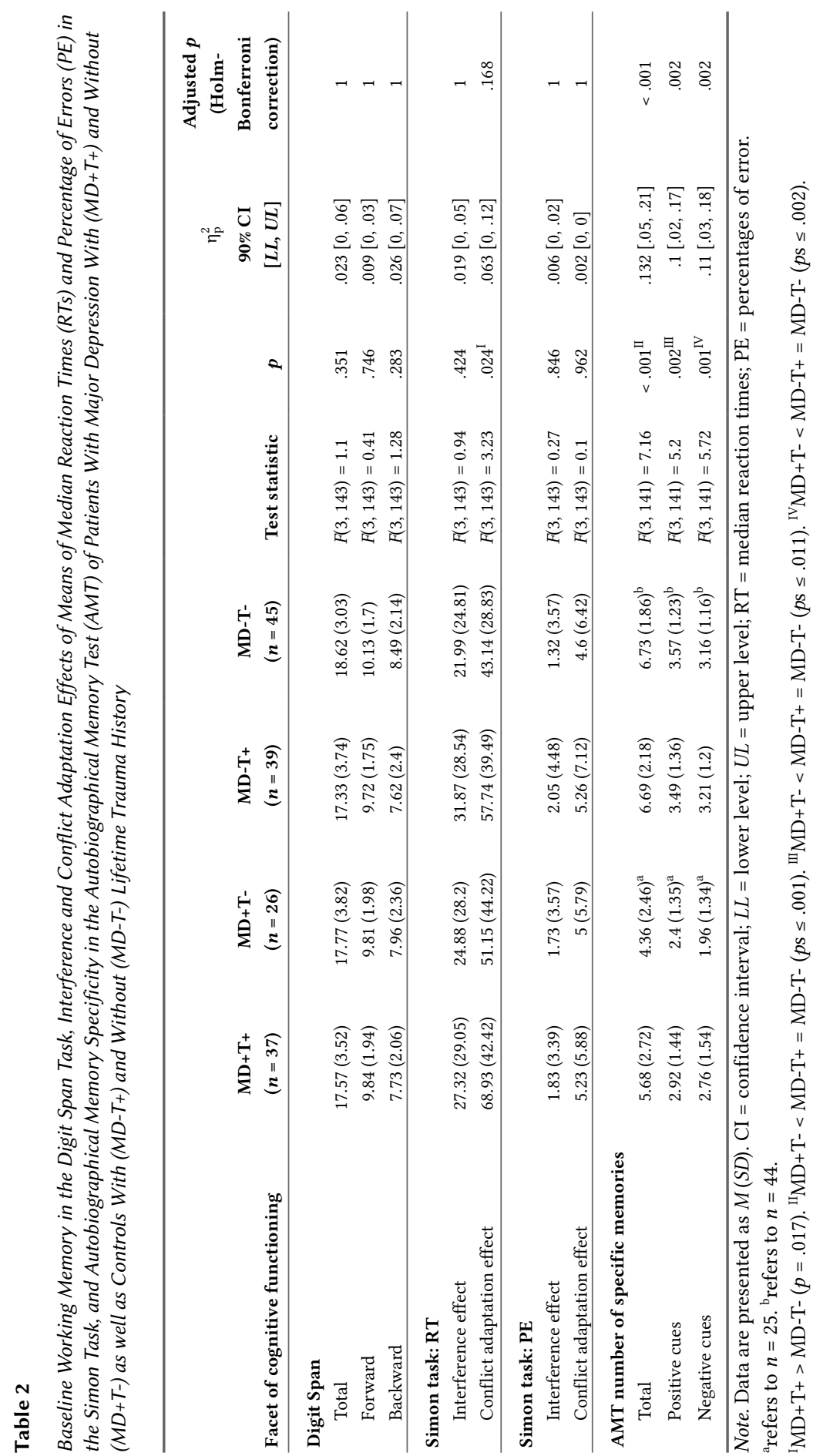




\section{Figure 1}

Mean $( \pm$ SEM) (A) Conflict Adaptation of Median RTs (Simon Task) and (B) Specificity of Autobiographical Memory (Autobiographical Memory Test) of Patients With Major Depression With $\left(M D+T_{+}\right)$and Without $\left(M D+T_{-}\right)$ as well as Controls With (MD-T+) and Without (MD-T-) Lifetime Trauma History at Baseline
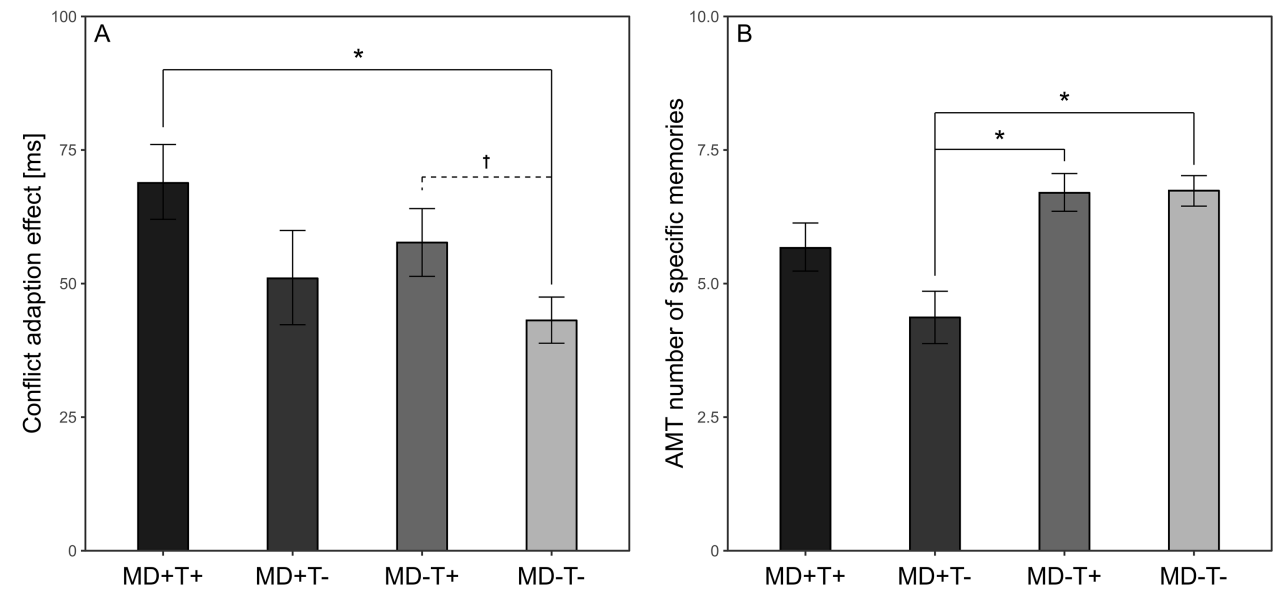

Note. ${ }^{*} p<.05, \dagger p<.10$, dotted lines indicate differentiating results between general linear and robust models.

\section{Clinical and Cognitive Treatment Outcome Under Consideration of Lifetime Trauma History}

$\mathrm{MD}+\mathrm{T}+(n=21), \mathrm{MD}+\mathrm{T}-(n=16)$, and MD-T- participants $(n=34)$ available for longitudinal analyses did not differ regarding baseline demographic/health-related characteristics (all $p s \geq .136$, see Supplementary Materials), except for higher medication intake in both MD groups $\left(\mathrm{X}_{2}^{2}=13.9, p=.001\right)$. However, including it as a covariate did not affect the longitudinal results. $\mathrm{MD}+\mathrm{T}+$ individuals reported a higher number of DSM-IV A1 traumatic events $(T H Q)$ than $\mathrm{MD}+\mathrm{T}$ - ones, which, in turn, reported more than MD-Tindividuals (all ps $\leq .036$ ). With respect to their frequency $(T H Q)$, as well as for childhood maltreatment severity $(C T Q)$, both $\mathrm{MD}+$ groups scored higher than the MD-T- one (all ps $\leq .035$ and all $p s \leq .002$, respectively).

Notably, while CBT led to substantial clinical improvements, MD+T+ and MD+Tindividuals did not differ regarding depressive symptom changes (BDI-II), subjectively evaluated therapy effects $(V E V-R)$, and percentage of dropouts (all $p s \geq .605$, see Table 3 ). Furthermore, no cognitive improvements over CBT in the clinical groups emerged (all $p s \geq .272$, see Table 3 ). However, for digit span, medium-to-large time effects indicated better performance at follow-up over all groups (all ps $\leq .009$ ). Robust regressions yielded similar results.

Regression analyses on the predictive value of lifetime trauma history (yes/no) for therapy outcome (BDI-II changes of depressive symptom severity and dropout status, 


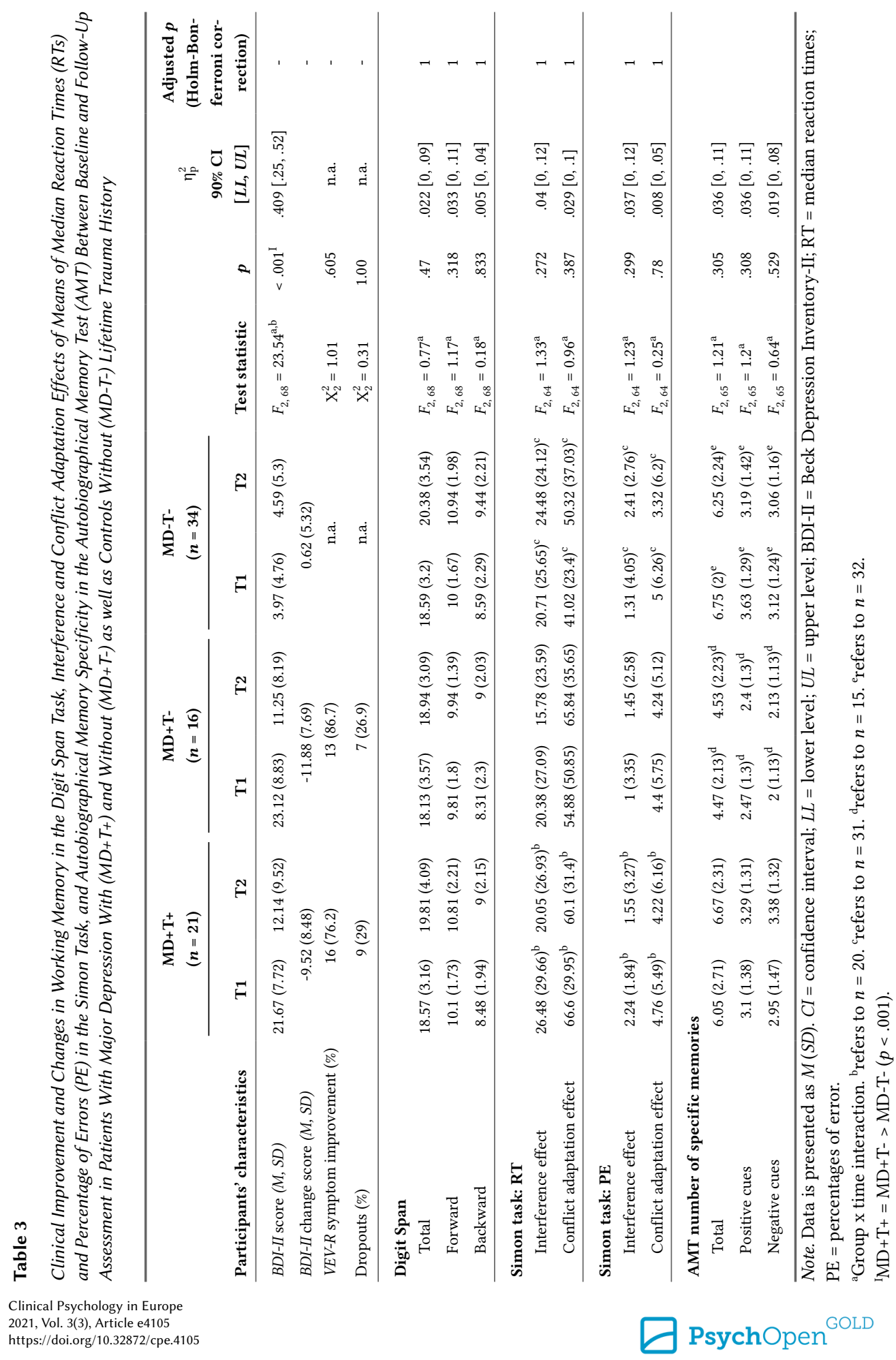


respectively) yielded no associations (all ps $\geq .391$ ). When, in a second step, adding respective facets of baseline cognitive functioning, more pronounced reductions of depressive symptom severity (BDI-II) emerged with smaller forward digit span, $b=1.48$, $95 \%$ CI $[0.07 ; 2.90], p=.041)$, while for all other measures of cognitive functioning, no predictive value emerged (all $p s \geq .059$ ). Adding, in a third step, interaction terms of lifetime trauma history (yes/no) and baseline cognitive functioning did not predict CBT outcome regarding $B D I-I I$ and dropout status (all $p s \geq .058$ ). Notably, robust regressions led to similar results.

\section{Discussion}

The aim of the study was to assess associations of (i) lifetime trauma history according to the DSM-IV (American Psychiatric Association, 2007) and (ii) facets of cognitive functioning (i.e., WM, interference susceptibility, conflict adaptation, and OGM) as well as (iii) their interaction with CBT outcome among patients with MD. At baseline, more pronounced conflict adaptation emerged in individuals with MD and lifetime trauma history in contrast to non-exposed healthy controls, while autobiographical memory was found to be primarily affected in MD without lifetime trauma history compared to both control groups. Notably, individuals with MD with and without lifetime trauma history did not differ regarding treatment outcome, and the cognitive parameters proved stable over CBT. Exploratory analyses suggested no direct or interacting association of lifetime trauma history, and only a tentative one of forward digit span, but no other aspects of cognitive functioning with treatment outcome.

\section{Baseline Cognitive Functioning}

On a cross-sectional level, the results support the role of lifetime trauma history for cognitive functioning in MD. While no differences emerged for interference susceptibility and WM, MD+T+ patients showed higher conflict adaptation of median RTs than MD-T- participants, with MD+T- and MD-T+ in between. This corresponds with previous findings from our group of more pronounced conflict adaptation in traumatized individuals with and possibly also without PTSD (Schindler et al., 2020; Steudte-Schmiedgen et al., 2014). However, as there also are suggested associations of conflict adaptation and depressive symptom severity (van Steenbergen et al., 2012), albeit without considering trauma history, further studies are desirable.

Interestingly, autobiographical memory yielded contrasting findings: $\mathrm{MD}+\mathrm{T}$ - patients showed more pronounced OGM compared to the healthy control groups, corresponding with our previous findings of OGM in PTSD, but not trauma exposure per se (Schindler et al., 2020), and suggestions from reviews and meta-analyses (Moore \& Zoellner, 2007; Ono et al., 2016; Sumner et al., 2010; Williams et al., 2007). Further, it supports the findings of 
Kuyken, Howell, and Dalgleish (2006) of OGM only in MD without (childhood) trauma history, but not those of Aglan et al. (2010) of OGM in MD with history of CSA. In sum, neither the results for conflict adaptation, nor those for OGM speak for a mere additive effect of trauma and MD on cognitive functioning, but rather for complex patterns with different impacts on different processes, and, potentially, different implications for clinical practice.

\section{Clinical and Cognitive Treatment Outcome Under Consideration of Lifetime Trauma History}

In contrast to several previous studies particularly on childhood trauma (reviewed in Nemeroff, 2016; Teicher \& Samson, 2013), our data suggest CBT to be equally effective in individuals with MD with and without the history of at least one traumatic event according to the DSM-IV. Several aspects may contribute to this divergence. Firstly, it is plausible that lifetime trauma, as examined in this study, does exert different effects than childhood trauma. Importantly, in our study, $\mathrm{MD}+\mathrm{T}+$ and $\mathrm{MD}+\mathrm{T}-$ groups reported equal $C T Q$ childhood maltreatment severity, and it is conceivable that this may have contributed to lacking group differences with respect to CBT effectiveness. Notably, also with respect to the $T H Q$, the $\mathrm{MD}+\mathrm{T}+$ and the $\mathrm{MD}+\mathrm{T}$ - groups did only differ on a descriptive level. However, it is important to consider that this instrument refers to the number and frequency of potentially traumatic events, for which the presence of the complete $D S M-I V$ criteria are not checked. In order to better understand the role of childhood and adulthood trauma for CBT effectiveness, studies explicitly contrasting individuals with MD (i) without lifetime trauma, (ii) with exclusively childhood, and (iii) with exclusively adulthood trauma as defined by the current diagnostic criteria are necessary. Furthermore, treatment differences might have played a role. Most prominently, the majority of studies reporting similar therapy outcome for MD with and without (particularly childhood) trauma history had applied combined psychotherapy and antidepressant medication (Lewis et al., 2010; Miniati et al., 2010; Nemeroff et al., 2003; but Asarnow et al., 2009), as was the case for approximately half of our sample. Further, we cannot rule out whether, in our study, trauma status had led to slight individual treatment adaptations by the responsible therapists. This might, for instance, have led to combined modifications of trauma-related and -unrelated automatic thought patterns, or the encouraging of restarting activities avoided after the trauma during behavioral interventions within the context of the utilized CBT manuals (Hautzinger, 1998, 2008). Thus, future studies applying more strictly manualized CBT and investigating larger MD groups with and without medication intake are required.

Additionally, the results corroborate previous findings of cognitive alterations in MD being highly stable over CBT (reviewed in Köhler et al., 2015; Moore \& Zoellner, 2007; Snyder \& Hankin, 2019), and of this to be irrespective of trauma history. While WM improved from baseline to follow-up, this is presumably attributable to practice/habituation 
effects, as it also concerned MD-T- individuals. As cognitive impairments are assumed to be associated with worse psychosocial functioning and increased relapse risk in MD (Rock et al., 2014), the continuous finding of this to not be adequately addressed by CBT shows the necessity to strive for "cognitive", next to clinical remission in MD (Bernhardt et al., 2019; Bortolato et al., 2016). For example, this might be achieved by directly targeting cognitive functioning during MD-centered CBT. While research on EF training in MD is still in its infancy (for a meta-analysis, see, e.g., Motter et al., 2016), there are promising results that OGM, as well as MD symptomatology itself may be influenced by interventions directly focusing on autobiographical recall, albeit with long-term stability still questionable (for a meta-analysis, see Barry, Sze, \& Raes, 2019).

The exploratory predictive analyses on lifetime trauma history and cognitive functioning for CBT do not provide clear results from which robust next steps could be derived. What can be clearly stated as of now is that there, again, was no evidence for a relevant role of lifetime trauma history. Further, only a singular association with cognitive parameters emerged, suggesting smaller WM to be associated with more pronounced depressiveness-related CBT effects. In sum, this pattern, albeit stemming from a very small sample size, supports the findings of Goodkind et al. (2016) on interference susceptibility, but stands at variance with those of Sumner et al. (2010) suggesting a predictive role of autobiographical memory specificity in MD. Future studies are needed to follow up on autobiographical memory in this context, or investigate whether other cognitive markers might be more suitable to predict clinical outcome after standardized psychotherapeutic/pharmacological treatment (e.g., Groves et al., 2018) with or without taking trauma history into account.

\section{Strengths, Limitations, and Outlook}

One central strength of the study is the naturalistic, highly ecologically valid study design. While the inclusion of a waiting control group of $\mathrm{MD}+\mathrm{T}+\mathrm{MD}+\mathrm{T}-$ patients not receiving CBT was impossible for ethical reasons, the fact that a healthy control group was studied longitudinally alongside the MD individuals is a further major strength, as it allowed the separation of CBT-associated and mere practice effects on cognitive functioning. However, limitations resulting from the naturalistic design are the heterogeneous manifestations of psychopathology and medication and the group differences in educational status and smoking. Further limitations include the lack of an objective, observer-rated outcome of depressiveness (e.g., the Hamilton Rating Scale for Depression; Hamilton, 1960), as well as the small sample sizes and the thus reduced statistical power for detecting especially interactive relationships. However, the fact that the vast majority of associations were confirmed in robust analyses corroborates the validity of the findings. Finally, behavioral tasks established in cognitive psychology, such as the ones used in our study, are characterized by task impurity, which describes the impossibility of assessing "pure" cognitive processes without simultaneously eliciting others (Miyake 
et al., 2000; e.g., Scott et al., 2015). In order to maximize transparency in data reporting, we chose to report subscale scores of the cognitive tasks for which different properties are discussed (Botvinick et al., 2004; Wechsler, 1997; Williams \& Broadbent, 1986). In addition, we acknowledge that for any of the assessed tasks, additional cognitive processes such as processing speed, attention, and motivation - while not directly studied - are inevitably involved.

\section{Conclusions}

In conclusion, the study is the first to examine lifetime trauma history, cognitive functioning, and their interaction in the context of CBT in patients with MD. On a cross-sectional level, conflict adaptation and autobiographical memory specificity emerged to be differentially affected in MD with and without lifetime trauma history. Contrary to previous research on childhood trauma, we found no evidence for a differential treatment response in patients with MD with and without lifetime trauma history as defined by the $D S M-I V$. Further, the cognitive parameters were stable over CBT, and only a singular predictive association of forward digit span, but no other facets of baseline cognitive functioning, lifetime trauma history, or their interaction with treatment outcome emerged. These insights into the interaction between lifetime trauma history and cognitive functioning provide unique extensions for research on MD psychopathology and treatment and underline the relevance of "cognitive" remission (Bernhardt et al., 2019; Bortolato et al., 2016). For achieving this aim, further research is required to allow more profound, neuroscience-informed diagnostic processes and personalized, multi-modal treatment approaches depending on patients' individual manifestation of cognitive functioning (De Raedt, 2020). 
Funding: This work was supported by the German Research Foundation (DFG; Grant Nos. STA 1213/5-1 to T.S. and J.H. and SFB 940/1 Project No. B5 to F.P. and C.K). L.S. was supported by the German Academic Scholarship Foundation. S.S.-S. was funded by a habilitation fellowship for women from the Faculty of Medicine Carl Gustav Carus, Technische Universität Dresden.

Acknowledgments: The authors would like to thank Elisabeth Cohors-Fresenborg, Fanny Weber, Anna-Katharina Richter, Juliane Kant, Inger-Sophie Hellerhoff, Kristin Werzner, the staff of the outpatient unit, and the members of the endocrinology laboratory of the Department of Psychology at the Technische Universität Dresden for their great help in conducting this research.

Competing Interests: All authors have no conflicts of interest to disclose.

Author Contributions: Lena Schindler: Formal analysis, data curation, writing- Original draft preparation, visualization. Tobias Stalder: Conceptualization, writing- Review \& Editing, funding acquisition. Clemens

Kirschbaum: Resources, writing- Review \& Editing. Franziska Plessow: Conceptualization, methodology, writingReview \& Editing. Sabine Schönfeld: Conceptualization, methodology, writing- Review \& Editing. Jürgen Hoyer: Resources, writing- Review \& Editing, funding acquisition. Sebastian Trautmann: Formal analysis, writing- Review \& Editing. Kerstin Weidner: Writing- Review \& Editing. Susann Steudte-Schmiedgen: Conceptualization, methodology, investigation, formal analysis, writing- Review \& Editing, supervision

\section{Supplementary Materials}

The Supplementary Materials contain the following items (for access see Index of Supplementary Materials below):

- Supplement 1 (Lifetime trauma history and mental disorder comorbidities)

- Supplement 2 (Baseline demographic, health-related, and clinical characteristics of patients with major depression with $(\mathrm{MD}+\mathrm{T}+)$ and without $(\mathrm{MD}+\mathrm{T}-)$ as well as controls without (MD-T-) lifetime trauma history available for longitudinal analyses)

\section{Index of Supplementary Materials}

Schindler, L., Stalder, T., Kirschbaum, C., Plessow, F., Schönfeld, S., Hoyer, J., Trautmann, S., Weidner, K., \& Steudte-Schmiedgen, S. (2021). Supplementary materials to "Lifetime trauma history and cognitive functioning in major depression and their role for cognitive-behavioral therapy outcome" [Additional information]. PsychOpen GOLD.

https://doi.org/10.23668/psycharchives.5073 


\section{References}

Aglan, A., Williams, J. M. G., Pickles, A., \& Hill, J. (2010). Overgeneral autobiographical memory in women: Association with childhood abuse and history of depression in a community sample. British fournal of Clinical Psychology, 49(3), 359-372. https://doi.org/10.1348/014466509X467413

Ajilchi, B., Nejati, V., Town, J. M., Wilson, R., \& Abbass, A. (2016). Effects of intensive short-term dynamic psychotherapy on depressive symptoms and executive functioning in major depression. The fournal of Nervous and Mental Disease, 204(7), 500-505. https://doi.org/10.1097/NMD.0000000000000518

American Psychiatric Association. (2007). Diagnostic and statistical manual of mental disorders: $D S M-I V-T R$ (4th ed., text revision, 10th print). American Psychiatric Association.

American Psychiatric Association. (2013). Diagnostic and statistical manual of mental disorders: DSM-5 (5th ed.). American Psychiatric Publishing.

Asarnow, J. R., Emslie, G., Clarke, G., Wagner, K. D., Spirito, A., Vitiello, B., Iyengar, S., Shamseddeen, W., Ritz, L., Birmaher, B., Ryan, N., Kennard, B., Mayes, T., De Bar, L., Mccracken, J., Strober, M., Suddath, R., Leonard, H., Porta, G., Keller, M., \& Brent, D. (2009). Treatment of selective serotonin reuptake inhibitor - Resistant depression in adolescents: Predictors and moderators of treatment response. Fournal of the American Academy of Child and Adolescent Psychiatry, 48(3), 330-339. https://doi.org/10.1097/CHI.0b013e3181977476

Barry, T. J., Sze, W. Y., \& Raes, F. (2019). A meta-analysis and systematic review of Memory Specificity Training (MeST) in the treatment of emotional disorders. Behaviour Research and Therapy, 116, 36-51. https://doi.org/10.1016/j.brat.2019.02.001

Barth, J., Munder, T., Gerger, H., Nüesch, E., Trelle, S., Znoj, H., Jüni, P., \& Cuijpers, P. (2013). Comparative efficacy of seven psychotherapeutic interventions for patients with depression: A network meta-analysis. PLoS Medicine, 10(5), Article e1001454.

https://doi.org/10.1371/journal.pmed.1001454

Beblo, T., Baumann, B., Bogerts, B., Wallesch, C.-W., \& Herrmann, M. (1999). Neuropsychological correlates of major depression: A short-term follow-up. Cognitive Neuropsychiatry, 4(4), 333-341. https://doi.org/10.1080/135468099395864

Bernhardt, M., Klauke, S., \& Schröder, A. (2019). Longitudinal course of cognitive function across treatment in patients with MDD: A meta-analysis. Journal of Affective Disorders, 249, 52-62. https://doi.org/10.1016/j.jad.2019.02.021

Bortolato, B., Miskowiak, K. W., Köhler, C. A., Maes, M., Fernandes, B. S., Berk, M., \& Carvalho, A. F. (2016). Cognitive remission: A novel objective for the treatment of major depression? BMC Medicine, 14, Article 9. https://doi.org/10.1186/s12916-016-0560-3

Botvinick, M. M., Braver, T. S., Barch, D. M., Carter, C. S., \& Cohen, J. D. (2001). Conflict monitoring and cognitive control. Psychological Review, 108(3), 624-652.

https://doi.org/10.1037/0033-295X.108.3.624

Botvinick, M. M., Cohen, J. D., \& Carter, C. S. (2004). Conflict monitoring and anterior cingulate cortex: An update. Trends in Cognitive Sciences, 8(12), 539-546.

https://doi.org/10.1016/j.tics.2004.10.003 
Cuijpers, P., Karyotaki, E., Weitz, E., Andersson, G., Hollon, S. D., \& van Straten, A. (2014). The effects of psychotherapies for major depression in adults on remission, recovery and improvement: A meta-analysis. Fournal of Affective Disorders, 159, 118-126.

https://doi.org/10.1016/j.jad.2014.02.026

Dannehl, K., Rief, W., \& Euteneuer, F. (2017). Childhood adversity and cognitive functioning in patients with major depression. Child Abuse \& Neglect, 70, 247-254.

https://doi.org/10.1016/j.chiabu.2017.06.013

De Raedt, R. (2020). Contributions from neuroscience to the practice of Cognitive Behaviour Therapy: Translational psychological science in service of good practice. Behaviour Research and Therapy, 125, Article 103545. https://doi.org/10.1016/j.brat.2019.103545

Diamond, A. (2013). Executive functions. Annual Review of Psychology, 64, 135-168. https://doi.org/10.1146/annurev-psych-113011-143750

Ehlers, A., Steil, R., Winter, H., \& Foa, E. (1996). Deutsche Übersetzung der Posttraumatic Stress Diagnostic Scale (PDS). Warneford Hospital, Department of Psychiatry.

Field, A. P., \& Wilcox, R. R. (2017). Robust statistical methods: A primer for clinical psychology and experimental psychopathology researchers. Behaviour Research and Therapy, 98, 19-38. https://doi.org/10.1016/j.brat.2017.05.013

Fischer, R., Dreisbach, G., \& Goschke, T. (2008). Context-sensitive adjustments of cognitive control: Conflict-adaptation effects are modulated by processing demands of the ongoing task. fournal of Experimental Psychology: Learning, Memory, and Cognition, 34(3), 712-718. https://doi.org/10.1037/0278-7393.34.3.712

Gast, U., Rodewald, F., Benecke, H.-H., \& Driessen, M. (2001). CTQ - Childhood Trauma Questionnaire - deutsche Fassung. Unpublished Manuscript, Medizinische Hochschule Hannover, Hannover, Germany.

Goodkind, M. S., Gallagher-Thompson, D., Thompson, L. W., Kesler, S. R., Anker, L., Flournoy, J., Berman, M. P., Holland, J. M., \& O’Hara, R. M. (2016). The impact of executive function on response to cognitive behavioral therapy in late-life depression. International fournal of Geriatric Psychiatry, 31(4), 334-339. https://doi.org/10.1002/gps.4325

Groves, S. J., Douglas, K. M., \& Porter, R. J. (2018). A systematic review of cognitive predictors of treatment outcome in major depression. Frontiers in Psychiatry, 9, Article 382.

https://doi.org/10.3389/fpsyt.2018.00382

Hager, W., \& Hasselhorn, M. (1994). Handbuch deutschsprachiger Wortnormen. Hogrefe.

Hamilton, M. (1960). A rating scale for depression. fournal of Neurology, Neurosurgery, and Psychiatry, 23, 56-62. https://doi.org/10.1136/jnnp.23.1.56

Hans, E., \& Hiller, W. (2013). Effectiveness of and dropout from outpatient cognitive behavioral therapy for adult unipolar depression: A meta-analysis of nonrandomized effectiveness studies. fournal of Consulting and Clinical Psychology, 81(1), 75-88. https://doi.org/10.1037/a0031080

Hautzinger, M. (1998). Depression (Fortschritte der Psychotherapie, Bd. 4). Hogrefe.

Hautzinger, M. (2008). Kognitive Verhaltenstherapie bei Depressionen: Behandlungsanleitungen und Materialien (Materialien für die klinische Praxis, Bd. 6., neu bearb. Aufl. [Nachdr.]). Beltz PVU. 
Hautzinger, M., Keller, F., \& Kühner, C. (2006). Beck Depressions-Inventar (BDI-II). Revision. Harcourt Test Services.

Holm, S. (1979). A simple sequentially rejective multiple test procedure. Scandinavian fournal of Statistics, 6(2), 65-70.

Hooper, L. M., Stockton, P., Krupnick, J. L., \& Green, B. L. (2011). Development, use, and psychometric properties of the Trauma History Questionnaire. Fournal of Loss and Trauma, 16(3), 258-283. https://doi.org/10.1080/15325024.2011.572035

Köhler, C. A., Carvalho, A. F., Alves, G. S., McIntyre, R. S., Hyphantis, T. N., \& Cammarota, M. (2015). Autobiographical memory disturbances in depression: A novel therapeutic target? Neural Plasticity, 2015, Article 759139. https://doi.org/10.1155/2015/759139

Kolassa, I.-T., \& Elbert, T. (2007). Structural and functional neuroplasticity in relation to traumatic stress. Current Directions in Psychological Science, 16(6), 321-325. https://doi.org/10.1111/j.1467-8721.2007.00529.x

Kuyken, W., Howell, R., \& Dalgleish, T. (2006). Overgeneral autobiographical memory in depressed adolescents with, versus without, a reported history of trauma. fournal of Abnormal Psychology, 115(3), 387-396. https://doi.org/10.1037/0021-843X.115.3.387

Lahr, D., Beblo, T., \& Hartje, W. (2007). Cognitive performance and subjective complaints before and after remission of major depression. Cognitive Neuropsychiatry, 12(1), 25-45. https://doi.org/10.1080/13546800600714791

Lewis, C. C., Simons, A. D., Nguyen, L. J., Murakami, J. L., Reid, M. W., Silva, S. G., \& March, J. S. (2010). Impact of childhood trauma on treatment outcome in the treatment for adolescents with depression study (TADS). Journal of the American Academy of Child and Adolescent Psychiatry, 49(2), 132-140. https://doi.org/10.1016/j.jaac.2009.10.007

Maercker, A. (2002). THQ - Trauma History Questionnaire - deutsche Fassung. Unpublished Manuscript, Universität Zürich, Zürich, Switzerland.

McIntyre, R. S., Cha, D. S., Soczynska, J. K., Woldeyohannes, H. O., Gallaugher, L. A., Kudlow, P., Alsuwaidan, M., \& Baskaran, A. (2013). Cognitive deficits and functional outcomes in major depressive disorder: Determinants, substrates, and treatment interventions. Depression and Anxiety, 30(6), 515-527. https://doi.org/10.1002/da.22063

Miniati, M., Rucci, P., Benvenuti, A., Frank, E., Buttenfield, J., Giorgi, G., \& Cassano, G. B. (2010). Clinical characteristics and treatment outcome of depression in patients with and without a history of emotional and physical abuse. Journal of Psychiatric Research, 44(5), 302-309. https://doi.org/10.1016/j.jpsychires.2009.09.008

Miyake, A., Emerson, M. J., \& Friedman, N. P. (2000). Assessment of executive functions in clinical settings: Problems and recommendations. Seminars in Speech and Language, 21(2), 169-183. https://doi.org/10.1055/s-2000-7563

Moore, S. A., \& Zoellner, L. A. (2007). Overgeneral autobiographical memory and traumatic events: An evaluative review. Psychological Bulletin, 133(3), 419-437.

https://doi.org/10.1037/0033-2909.133.3.419 
Motter, J. N., Pimontel, M. A., Rindskopf, D., Devanand, D. P., Doraiswamy, P. M., \& Sneed, J. R. (2016). Computerized cognitive training and functional recovery in major depressive disorder: A meta-analysis. Journal of Affective Disorders, 189, 184-191. https://doi.org/10.1016/j.jad.2015.09.022

Nanni, V., Uher, R., \& Danese, A. (2012). Childhood maltreatment predicts unfavorable course of illness and treatment outcome in depression: A meta-analysis. The American fournal of Psychiatry, 169(2), 141-151. https://doi.org/10.1176/appi.ajp.2011.11020335

Nemeroff, C. B. (2016). Paradise lost: The neurobiological and clinical consequences of child abuse and neglect. Neuron, 89(5), 892-909. https://doi.org/10.1016/j.neuron.2016.01.019

Nemeroff, C. B., Heim, C. M., Thase, M. E., Klein, D. N., Rush, A. J., Schatzberg, A. F., Ninan, P. T., McCullough, J. P., Jr., Weiss, P. M., Dunner, D. L., Rothbaum, B. O., Kornstein, S., Keitner, G., \& Keller, M. B. (2003). Differential responses to psychotherapy versus pharmacotherapy in patients with chronic forms of major depression and childhood trauma. Proceedings of the National Academy of Sciences of the United States of America, 100(24), 14293-14296. https://doi.org/10.1073/pnas.2336126100

Oberg, A. L., \& Mahoney, D. W. (2007). Linear mixed effects models. Methods in Molecular Biology, 404, 213-234. https://doi.org/10.1007/978-1-59745-530-5_11

Ono, M., Devilly, G. J., \& Shum, D. H. K. (2016). A meta-analytic review of overgeneral memory: The role of trauma history, mood, and the presence of posttraumatic stress disorder. Psychological Trauma: Theory, Research, Practice, and Policy, 8(2), 157-164. https://doi.org/10.1037/tra0000027

Peeters, F., Wessel, I., Merckelbach, H., \& Boon-Vermeeren, M. (2002). Autobiographical memory specificity and the course of major depressive disorder. Comprehensive Psychiatry, 43(5), 344-350. https://doi.org/10.1053/comp.2002.34635

$R$ [Computer software]. (2017). R Foundation for Statistical Computing. Vienna, Austria. Rock, P. L., Roiser, J. P., Riedel, W. J., \& Blackwell, A. D. (2014). Cognitive impairment in depression: A systematic review and meta-analysis. Psychological Medicine, 44(10), 2029-2040. https://doi.org/10.1017/S0033291713002535

Royall, R. M. (1986). Model robust confidence intervals using maximum likelihood estimators. International Statistical Review / Revue Internationale De Statistique, 54(2), 221-226. https://doi.org/10.2307/1403146

Saleh, A., Potter, G. G., McQuoid, D. R., Boyd, B., Turner, R., MacFall, J. R., \& Taylor, W. D. (2017). Effects of early life stress on depression, cognitive performance and brain morphology. Psychological Medicine, 47(1), 171-181. https://doi.org/10.1017/S0033291716002403

Schindler, L., Stalder, T., Kirschbaum, C., Plessow, F., Schönfeld, S., Hoyer, J., Trautmann, S., \& Steudte-Schmiedgen, S. (2020). Cognitive functioning in posttraumatic stress disorder before and after cognitive-behavioral therapy. Journal of Anxiety Disorders, 74, Article 102265. https://doi.org/10.1016/j.janxdis.2020.102265 
Schmid, M., \& Hammar, A. (2013). A follow-up study of first episode major depressive disorder: Impairment in inhibition and semantic fluency-potential predictors for relapse? Frontiers in Psychology, 4, Article 633. https://doi.org/10.3389/fpsyg.2013.00633

Schönfeld, S., \& Ehlers, A. (2006). Overgeneral memory extends to pictorial retrieval cues and correlates with cognitive features in posttraumatic stress disorder. Emotion, 6(4), 611-621. https://doi.org/10.1037/1528-3542.6.4.611

Scott, J. C., Matt, G. E., Wrocklage, K. M., Crnich, C., Jordan, J., Southwick, S. M., Krystal, J. H., \& Schweinsburg, B. C. (2015). A quantitative meta-analysis of neurocognitive functioning in posttraumatic stress disorder. Psychological Bulletin, 141(1), 105-140. https://doi.org/10.1037/a0038039

Sheehan, D. V., Lecrubier, Y., Sheehan, K. H., Amorim, P., Janavs, J., Weiller, E., Hergueta, T., Baker, R., \& Dunbar, G. C. (1998). The Mini-International Neuropsychiatric Interview (M.I.N.I.): The development and validation of a structured diagnostic psychiatric interview for DSM-IV and ICD-10. The fournal of Clinical Psychiatry, 59(Suppl 20), 22-33.

Sherin, J. E., \& Nemeroff, C. B. (2011). Post-traumatic stress disorder: The neurobiological impact of psychological trauma. Dialogues in Clinical Neuroscience, 13(3), 263-278. https://doi.org/10.31887/DCNS.2011.13.2/jsherin

Simon, J. R. (1990). The effects of an irrelevant directional cue on human information processing. In R. W. Proctor \& T. G. Reeve (Eds.), Stimulus-response compatibility: An integrated perspective (pp. 31-86). Elsevier.

Snyder, H. R. (2013). Major depressive disorder is associated with broad impairments on neuropsychological measures of executive function: A meta-analysis and review. Psychological Bulletin, 139(1), 81-132. https://doi.org/10.1037/a0028727

Snyder, H. R., \& Hankin, B. L. (2019). Cognitive function in mood and anxiety disorders. In R. A. Stern \& M. L. Alosco (Eds.), The Oxford handbook of adult cognitive disorders (pp. 249-273). Oxford University Press.

Stata Statistical Software: Release 15 [Computer software]. (2017). College Station, TX, USA. Steudte, S., Kirschbaum, C., Gao, W., Alexander, N., Schonfeld, S., Hoyer, J., \& Stalder, T. (2013). Hair cortisol as a biomarker of traumatization in healthy individuals and posttraumatic stress disorder patients. Biological Psychiatry, 74(9), 639-646. https://doi.org/10.1016/j.biopsych.2013.03.011

Steudte-Schmiedgen, S., Stalder, T., Kirschbaum, C., Weber, F., Hoyer, J., \& Plessow, F. (2014). Trauma exposure is associated with increased context-dependent adjustments of cognitive control in patients with posttraumatic stress disorder and healthy controls. Cognitive, Affective \& Behavioral Neuroscience, 14(4), 1310-1319. https://doi.org/10.3758/s13415-014-0299-2

Sumner, J. A., Griffith, J. W., \& Mineka, S. (2010). Overgeneral autobiographical memory as a predictor of the course of depression: A meta-analysis. Behaviour Research and Therapy, 48(7), 614-625. https://doi.org/10.1016/j.brat.2010.03.013 
Teicher, M. H., \& Samson, J. A. (2013). Childhood maltreatment and psychopathology: A case for ecophenotypic variants as clinically and neurobiologically distinct subtypes. The American Fournal of Psychiatry, 170(10), 1114-1133. https://doi.org/10.1176/appi.ajp.2013.12070957

van Steenbergen, H., Band, G. P. H., \& Hommel, B. (2010). In the mood for adaptation: How affect regulates conflict-driven control. Psychological Science, 21(11), 1629-1634.

https://doi.org/10.1177/0956797610385951

van Steenbergen, H., Booij, L., Band, G. P. H., Hommel, B., \& van der Does, A. J. W. (2012). Affective regulation of cognitive-control adjustments in remitted depressive patients after acute tryptophan depletion. Cognitive, Affective \& Behavioral Neuroscience, 12(2), 280-286. https://doi.org/10.3758/s13415-011-0078-2

Vasterling, J. J., \& Arditte Hall, K. A. (2018). Neurocognitive and information processing biases in posttraumatic stress disorder. Current Psychiatry Reports, 20(11), Article 99.

https://doi.org/10.1007/s11920-018-0964-1

Wechsler, D. (1997). Wechsler Memory Scale - Third Edition (WMS-III). The Psychological Corporation.

Williams, J. M. G., Barnhofer, T., Crane, C., Herman, D., Raes, F., Watkins, E., \& Dalgleish, T. (2007). Autobiographical memory specificity and emotional disorder. Psychological Bulletin, 133(1), 122-148. https://doi.org/10.1037/0033-2909.133.1.122

Williams, J. M. G., \& Broadbent, K. (1986). Autobiographical memory in suicide attempters. fournal of Abnormal Psychology, 95(2), 144-149. https://doi.org/10.1037/0021-843X.95.2.144

Wittchen, H.-U., \& Pfister, H. (1997). DIA-X-Interviews: Manual für Screening-Verfahren und Interview; Interviewheft Längsschnittuntersuchung (DIA-X-Lifetime); Ergänzungsheft (DIA-XLifetime); Interviewheft Querschnittuntersuchung (DIA-X-12Monate); Ergänzungsheft (DIA$X$-12Monate); PC-programm zur Durchführung des Interviews (Längs- und Querschnittuntersuchung); Auswertungsprogramm. Swets \& Zeitlinger.

Zielke, M., \& Kopf-Mehnert, C. (2001). Der VEV-R-2001: Entwicklung und testtheoretische Reanalyse der revidierten Form des Veränderungsfragebogens des Erlebens und Verhaltens (VEV). Praxis Klinische Verhaltensmedizin und Rehabilitation, 53, 7-19.

\section{EACLIPT}

Clinical Psychology in Europe (CPE) is the official journal of the European Association of Clinical Psychology and Psychological Treatment (EACLIPT).

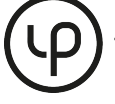
leibniz-psychology.org

PsychOpen GOLD is a publishing service by Leibniz Institute for Psychology (ZPID), Germany. 